Archives de sciences sociales des religions

137 | janvier - mars 2007

Varia

\title{
L'humanitarisme hindou ou la dévotion civile
}

\section{Pascale Lépinasse}

\section{OpenEdition}

Journals

Édition électronique

URL : http://journals.openedition.org/assr/4314

DOI : 10.4000/assr.4314

ISSN : $1777-5825$

Éditeur

Éditions de l'EHESS

Édition imprimée

Date de publication : 1 avril 2007

Pagination : 85-105

ISBN : 978-2-7132-2142-2

ISSN : 0335-5985

Référence électronique

Pascale Lépinasse, "L'humanitarisme hindou ou la dévotion civile», Archives de sciences sociales des religions [En ligne], 137 | janvier - mars 2007, mis en ligne le 05 juin 2010, consulté le 19 avril 2019. URL : http://journals.openedition.org/assr/4314; DOI : 10.4000/assr.4314 


\section{Pascale Lépinasse}

\section{L'humanitarisme hindou ou la dévotion civile}

La visibilité actuelle des gourous modernes représente un phénomène sans précédent sur la scène indienne. En effet, ces trente dernières années ont vu s'amplifier la médiatisation et la popularité de maîtres spirituels d'un nouveau type, à la fois bénéficiaires de la vague New Age des années 1970 et de l'émergence d'une classe moyenne indienne de plus en plus mobile et prospère. Leaders de mouvements religieux d'inspiration hindoue mais de dimension planétaire, ils excellent à substituer aux représentations familières du divin et aux pratiques cultuelles traditionnelles des voies de salut "à la carte », plus conformes aux attentes de populations vivant au rythme de la modernité urbaine et matérialiste. En plus du mariage de recettes de développement personnel, de références à une prétendue " Grande Tradition » sanskrite et d'un sentiment nationaliste plus ou moins larvé, l'argument humanitaire suscite les vocations spirituelles les plus surprenantes : la plupart des disciples sont manifestement conquis par l'engagement philanthropique de maitres au profil d'humanistes.

\section{Profil du " gourou flottant "}

L'autorité du gourou est, en Inde, coutumière, les maîtres religieux s'étant succédés, depuis l'époque médiévale, pour interpréter, voire renouveler, le corpus fondateur de "l'hindouisme ". Lui-même produit de l'interaction entre doctrines lettrées du brahmanisme ${ }^{1}$ et coutumes locales, l'hindouisme n'a cessé d'apparaître comme un chantier ouvert, offrant d'autant plus d'espace à la pluralité que toute reformulation à caractère exégétique peut inspirer à son auteur et ses partisans la création d'une nouvelle tradition sectaire, appelée en sanskrit sampradāya. Conformes aux normes fondatrices brahmaniques, et dépendantes d'une lignée de maîtres (paramparā) qui en garantit la valeur et l'honnêteté, les sampradāya signalent davantage la continuité dans la diversité de l'hindouisme que sa fracture.

1. Ce sont d'abord les textes du Véda, source d'autorité sacrée, que les brahmanes imposèrent au nord de l'Inde au milieu du deuxième millénaire avant Jésus-Christ. L'exégèse de ce corpus a produit, au cours des derniers siècles avant notre ère, des écritures plurielles et diversifiées, parfois en contradiction les unes avec les autres, mais toutes considérées comme vérités potentielles. Citons les Upanishad et les deux épopées du Ramayana et du Mahabharata. 
Leurs gourous fondateurs jouissent d'un crédit exceptionnel, soit parce qu'ils maîtrisent à la perfection les Écritures, soit parce que de formidables performances ascétiques les ont rendus capables de prodiges - certains conjuguant même ces deux talents. Par son savoir et son charisme, le maître attire à lui de multiples adeptes. Entre maître et disciples s'instaure une relation intime, d'abord exprimée par l'initiation (diksha) qui suppose que le gourou transmette au nouveau disciple la formule sacrée (mantra) propre à la paramparā dans laquelle il s'inscrit. Traditionnellement, la dīkshā exige la confidentialité, qu'elle implique l'entrée de l'adepte dans un ordre monastique, ou qu'elle n'exige, au contraire, nul retrait du monde. Or, les nouveaux gourous n'hésitent pas à transmettre le mantra publiquement à des assemblées d'une densité impressionnante ${ }^{2}$. La relation maître/disciple se voit dès lors «dé-privatisée ", débordée par la «démocratisation » du fait initiatique. Le degré d'autonomie affiché par la plupart des gourous modernes confirme qu'il n'est plus besoin de se référer à une paramparā pour faire autorité, beaucoup étant initialement des personnages laïques avant de devenir des icônes religieuses. Leur sainteté, comme leur ascendant, se fonde désormais sur de nouveaux appuis, que ce soit le soudain charisme né d'une révélation, l'accomplissement de miracles, ou le don de soi mû par une compassion «supra-humaine " ${ }^{3}$. Non plus dépositaires temporaires mais propriétaires permanents et exclusifs du savoir qu'ils détiennent, ils sont libres d'en disposer, en transgressant, par exemple, la loi du secret. Leur objectif n'est pas plus de perpétuer une paramparā ancienne que d'en créer une nouvelle, mais de fonder un mouvement. Aussi se consacrent-ils moins à leur succession qu'à l'urgente diffusion planétaire de leurs message et action, urgence à laquelle les réseaux modernes de communication s'avèrent mieux adaptés que le relais traditionnel de la paramparā. Indépendamment de la stratégie universaliste qu'elle représente, cette démarche invite à penser le gourou comme une individualité à part entière. Autorité unique, inégalable et irremplaçable, il fait volontiers figure de prophète ou de messie, et cesse d'être le simple intermédiaire dépourvu d'ego par lequel la transmission d'une sagesse s'opérait. Ce dernier point nous incite à fonder à son endroit l'expression de "gourou flottant».

Il s'agit là d'une rupture surprenante avec la norme. Nous sommes frappés de voir avec quelle assiduité le public interpellé valide l'effacement de la dimension ésotérique. La présentation d'un très populaire "ONG-isme », ainsi qu'une

2. Les médias et les institutions concernés évoquent régulièrement des foules de plusieurs milliers de personnes venant écouter les gourous les plus renommés, tels Ramdev, Asharam Ji, Amma, Sathya Sai Baba, ou encore Shri Shri Ravi Shankar.

3. À titre d'exemples, on vénère Amma parce que ses bras maternels étreignent sans fatigue apparente plusieurs milliers de personnes à chacune de ses «tournées " à travers le monde, S. Sai Baba parce qu'il déglutit des symboles shivaïtes et fait apparaître de la cendre à volonté, et S.S. Ravi Shankar parce qu'il a reçu la révélation d'un yoga du souffle aussi facile à pratiquer qu'efficace. À propos des deux premiers, consulter Maya WARrIER, Hindu Selves in a Modern World. Guru Faith in the Mata Amritanandamayi, Routledge Curzon, 2005, et Deborah SwALlow, "Ashes and powers. Myth, rite and miracle in an Indian god-man's cult ", Modern Asian Studies, 16, 1982. 
brève esquisse du profil de l'audience séduite, nous aideront à comprendre cette approbation. Nous ne devrons pas négliger l'important engouement de la diaspora qui conforte les partis pris de médiatisation et de mobilité des gourous flottants. Leur position à la tête d'institutions tentaculaires, bâties sur une logique de réseaux, les investit de responsabilités de gestionnaires, et la densité de leur emploi du temps leur fait endosser le rythme de vie trépidant « d'entrepreneurs », pour reprendre les termes dans lesquels Chantal Saint-Blancat définit les leaders charismatiques des mouvements de renouveau chrétien ${ }^{4}$.

Cette définition vaut pour les nouvelles figures d'autorité religieuse qui nous intéressent. La propension des gourous indiens au «bricolage », pour employer une métaphore courante de la sociologie religieuse occidentale, revêt sans conteste aujourd'hui une nouvelle dimension, que nos trois exemples entendent illustrer.

\section{Du sampradaya au nouveau mouvement religieux}

Le Bochasanwasi Shri Akshar Purushottam Samstha (BAPS), la Brahma Kumaris World Spiritual University (BKWSU) et The Art of Living (AoL), nés respectivement en 1906, 1937, et 1982, s'offrent comme les trois ponctuations « idéales ${ }^{5}$ d'une progression partant de l'orientation la plus traditionnellement définie (le BAPS) et aboutissant à la plus progressiste (AoL), en cheminant par une proposition hybride relativement baroque (la BKWSU). Concurrentes sur la scène indienne ${ }^{6}$, ces trois institutions ont quelque chose à nous dire du présent et de l'avenir de l'hindouisme, de ses adaptations et métamorphoses comme de ses continuités et résistances. Elles occupent une position ambiguë, refusant de se voir réduites à de simples traditions théologiques confinées au sol indien et alliant ancrage dans la tradition et postulat à l'universalisme. Une même motivation les anime, celle de répondre aux attentes de classes sociales inscrites dans la dynamique individualisante du profit, matériel et spirituel. Chacune entend parler le langage du progressisme et combattre l'obscurantisme, en valorisant l'intellectualisation du religieux, en faisant cas de la performance sociale, et en s'investissant d'une mission éducative à grande échelle. Toutes trois partagent le goût des nouvelles technologies afin d'optimiser la promotion et la diffusion de leur message, et entretiennent leur visibilité à grand renfort médiatique. Au-delà d'une volonté "d'éthiciser » l'hindouisme fort redevable au néo-vedanta du XIX ${ }^{e}$ siècle, elles

4. Chantal Saint-Blancat : «Les entrepreneurs religieux de la globalisation sont d'infatigables voyageurs et d'excellents utilisateurs du Cybernet. Experts en prospection de marché, confection et distribution de biens marchands symboliques, ils constituent de redoutables concurrents pour l'autorité religieuse traditionnelle » in J.-P. BASTIAN, K. Rousselet et F. CHAMPION, dirs., La globalisation du religieux, Paris, L'Harmattan, 2001, p. 81.

5. Presque au sens d'idéal-type wébérien.

6. En témoignent un même soin porté à leurs infrastructures frôlant le gigantisme, et leur présence rivale dans le secteur humanitaire. Sur le terrain d'une catastrophe (naturelle, terroriste, etc.), chacune prétend : "We were the first to come, the last to leave". 
prêchent en langage militant l'urgente re-moralisation de la civilisation indienne dans son ensemble, et, par extension, celle de l'humanité. Les fondements culturels et cultuels indiens, systématiquement et délibérément entendus au sens de « hindous », constituent à leur sens les outils les mieux appropriés à la diffusion universelle du Bien.

\section{Le doyen}

Le BAPS est le plus " orthodoxe" des trois mouvements étudiés, le seul à centrer sa pratique sur le culte rendu aux images dans l'espace traditionnel $\mathrm{du}$ temple. Même s'il ne s'en contente pas, il ne redoute pas l'appellation de sampradāya et, de fait, présente toutes les caractéristiques d'un regroupement sectaire classique de l'hindouisme : un noyau dur d'ascètes renonçants, nommés sādhu ou santos; une vaste assemblée d'adeptes laïques; des écritures consensuelles (essentiellement rédigées par le fondateur); un gourou conférant la diksha dans les règles et perpétuant une longue paramparā.

Le groupe inscrit sa doctrine et sa pratique spirituelles dans bhakti marga, la "voie de la dévotion ". Il relève d'une scission d'avec l'éminente tradition fondée par un yogi vishnouite, Swaminarayan (1780-1830). Renonçant à l'âge précoce de onze ans, ce dernier parcourut sa région natale de l'Andhra Pradesh jusqu'au Gujarat, dont l'état de dévastation (dû à une tragique succession de catastrophes naturelles et d'épidémies, et aux exactions anarchiques de chefs de bandes locaux) l'aurait convaincu de s'associer aux initiatives de restructuration menées par les Britanniques : tandis que le pouvoir colonial unifiait et centralisait le régime administratif, Swaminarayan, de son côté, s'appliquait à réformer les mœurs et pratiques des ordres religieux qu'il jugeait décadentes, hédonistes ou trop idolâtres, en particulier celles relevant du "tantrisme de la main gauche » et de la secte vallabhacari (cf. infra). Animé par un fort puritanisme, il exigeait de ses disciples une moralité irréprochable, et les incitait à se vouer à la philanthropie et au bien-être de leurs concitoyens à travers tout type de services sociaux ${ }^{7}$.

La stabilité du sampradaya vacilla en 1907, lorsqu'un santos dissident, Shastri Maharaj (1865-1951), réunit quelques partisans et créa sa propre communauté, le Bochasanwasi Shri Akshar Purushottam Sanstha. Ce dernier soutenait encore davantage l'éthique et l'engagement altruiste actif des santos, au point de conférer au BAPS une dimension missionnaire et militante, - pleinement assumée depuis l'acquisition de son statut d'ONG.

Basé à Ahmedabad (Gujarat), le BAPS revendique aujourd'hui un million de membres laïques et compte un peu plus de sept cents santos dans son ordre

7. Aujourd'hui encore, chaque novice ordonné ascète par son gourou s'engage à respecter cinq commandements : le célibat absolu, incluant jusqu'à la pensée même de l'autre féminin ; le renoncement aux sens, notamment au goût ; la rupture des liens familiaux ; la non-avidité, associée à la pauvreté ; l'absence d'ego. 
monastique, uniquement masculin. Il a gagné en importance sous la direction de Pramukh Swami, à la tête de l'institution depuis 1971. Si l'on ne peut tout à fait le qualifier de "flottant", le gourou de l'institution se distingue nettement de ses prédécesseurs par son charisme social. Il lui confère l'aura d'un infatigable et moderne saint sauveur, d'autant plus progressiste que Pramukh Swami s'est employé à développer une voie d'accès égalitaire et standardisée au renoncement. Les santos expérimentent, en effet, une période probatoire au sein d'une Sadhu Training School (école de formation des $s \bar{a} d h u$ ), qui les forme à une vie monastique autre que contemplative, passive ou individualiste. Les objectifs d'expansion et de promotion du BAPS les conduisent à conjuguer des savoir-faire psychologiques, manuels et scientifiques, des compétences en technologie de pointe et en marketing. Pramukh Swami conseille aux jeunes gens désirant l'ordination d'achever d'abord leur cursus universitaire avant de se présenter à l'initiation, s'assurant ainsi que tout santos a bénéficié à l'université d'une éducation occidentalisée. Se présentant comme une " organisation socioreligieuse », le BAPS entretient plusieurs départements voués à la recherche et met en œuvre divers types d'actions caritatives. L'ensemble repose sur le dynamisme et l'exemplaire dévouement de ses performants renonçants et l'actif support matériel des laïcs. Le recrutement constitue, de la sorte, la grande force du BAPS.

Demeurée indissociable de l'État côtier du Gujarat qui l'a vu naître, l'institution s'est d'abord distinguée par un fort ancrage régional. Elle ne touche plus seulement, aujourd'hui, la population locale, mais l'impressionnante diaspora gujarati répartie aux quatre coins du globe. Londres représente le deuxième centre du BAPS après Ahmedabad et, depuis 1995, abrite le plus grand temple indien d'Occident, Neasden, prévu pour accueillir trois mille dévots. Le BAPS se montre inquiet du devenir de la communauté gujarati expatriée, estimant qu'elle s'expose à quantité de périls encourus par quiconque côtoie "l'étranger ». En plus de la présence des santos, depuis peu résidents permanents du temple londonien, Pramukh Swami mène sans relâche de longues tournées mondiales afin de prêcher à ses disciples laïques l'isolationnisme susceptible de les protéger des influences néfastes qui émaneraient de l'Occident : il recommande d'éviter tout contact avec les médias - cinéma, littérature ou encore télévision -, et invite à respecter certaines règles disciplinaires, notamment le végétarisme et la sobriété, l'étude régulière des Écritures, la vénération quotidienne de l'image de Swaminarayan (puja et darshana) et le service inconditionné du maître. Tels sont les piliers d'une éthique devenue subrepticement morale. La confusion entre culture indienne et culture hindoue est patente. Dangereux amalgame, qui affaiblit la volonté universaliste dont fait preuve le BAPS à travers ses ambitions humanitaires.

Paradoxalement, ces injonctions côtoient des recettes de réalisation personnelle issues d'une pensée positive... typiquement anglo-saxonne ! Le dépassement de soi, l'opportunisme professionnel, la performance technique au service du prosélytisme constituent des valeurs de plus en plus reconnues par la littérature du 
groupe. La lecture de Bliss, mensuel en langue anglaise édité par le BAPS, offre au lecteur une alternance intéressante entre des articles d'esprit plutôt « orthodoxe » et " éthiquement corrects » (extraits hagiographiques de Swaminarayan et de renonçants exemplaires, fables puritaines et anecdotes moralisatrices, etc.), et des chroniques d'esprit New Age. Anthony Robbins ${ }^{8}$ fait régulièrement figure de modèle, afin de repenser les voies du bien-être en termes de stratégies menant au pouvoir.

\section{La cadette}

À l'origine de la BKWSU se trouve Dada Lekhraj (1872-1969), riche diamantaire d'obédience vishnouite qui, la soixantaine venue, se préoccupa de régénérer l'hindouisme. Il recommandait d'en extraire les "scories" responsables de la régression de l'admirable religion de l'antiquité indienne en un ensemble de superstitions et pratiques idolâtres et avilissantes! Selon lui, polythéisme, culte des images et vénération des gourous excluaient l'Inde de la modernité, et la privaient de reconnaissance sur la scène mondiale. Originaire de Hyderabad (Sindh), Dada Lekhraj y fonda en 1937 «Om Mandali », cercle spirituel rassemblant des individus autour de la lecture de la Bhagavad Gita afin d'y retrouver l'essence de l'hindouisme. Cette société ne constituerait rien d'autre qu'une expérimentation néo-hindoue de plus, si Lekhraj ne l'avait créée, d'une part, sous le choc d'une révélation de nature millénariste, d'autre part, à destination d'un auditoire prioritairement féminin. Bhakta modéré, Lekhraj eut, un jour, la vision des dieux Shiva et Vishnou, lui révélant l'imminente apocalypse de l'univers à travers des vues d'épouvante, suivies d'images enchanteresses de l'éden qui succéderait à la destruction, à condition que l'humanité survivante possédât les qualités nécessaires à son avènement. Lekhraj délaissa aussitôt ses florissantes affaires pour embrasser la carrière de gourou flottant sous le nom de Brahma Baba, et susciter ladite humanité.

La BKWSU tient ses quartiers sur le Mont Abu, au Rajasthan depuis 1951, mais connaît une implantation solide dans toutes les villes importantes du souscontinent indien. En choisissant de se rebaptiser "université », elle évite de se voir limitée à un contexte géographique, religieux et culturel. De fait, l'institution prétend délivrer un enseignement basé sur un programme d'étude rationnel, c'està-dire planifié et progressif, et présente ses adeptes comme des étudiants et non comme des disciples... même si tous sont «BK» $\left(\right.$ Brahma Kumari $\left.{ }^{9}\right)$ de Brahma Baba.

8. Un physique avantageux, des moyens considérables, une pensée positive archétypale, ce chamane des temps modernes est le winner par excellence. Il organise des shows auxquels assistent des milliers de personnes, qu'il « électrise » au point de les faire marcher sur les braises, afin de les convaincre qu'il les hissera au pinacle du succès.

9. La traduction littérale en est " fille-vierge de Brahma ». 
Le « cursus » vise à l'acquisition de techniques d'autodivinisation, afin que les BK restaurent l'âge d'or attendu. Tout adepte, qu'il soit totalement dédié à l'institution (il réside alors dans l'un des centres, exerce rarement une activité professionnelle, et n'a pas de vie familiale), ou non, se voit imposer les mêmes proscriptions et prescriptions. Il se voue à un ensemble de disciplines rassemblées sous le nom de raja yoga (yoga royal), alliance de méditation intensive et de pratique sévère de la chasteté qui doivent à terme l'insensibiliser à la pulsion : détourné de ses satisfactions personnelles, le pratiquant abstinent jouit d'une disponibilité qui l'autorise à développer un niveau de conscience supérieur. Ses qualités supra-humaines l'orientent vers le chemin de l'altruisme et du Bien universel, lui confiant la mission de perfectionner le monde. C'est au nom de ce système de cause à effet que la BKWSU jouit, depuis 1982, du statut consultatif auprès de l'ONU en tant qu'ONG.

Brahma Baba fustigeait la soumission sexuelle à laquelle le varna-ashramadharma ${ }^{10}$ condamnait la femme, et regardait la chasteté comme une arme d'émancipation. Il attribuait aux femmes d'éminentes qualités de cœur, leur conférant un rôle clef dans l'établissement du futur âge d'or : il leur revient aujourd'hui d'incarner la dimension philanthropique de la BKWSU. Ainsi, depuis la disparition du fondateur, l'organisation ne connaît pas de gourou mais dépend d'un directoire de dadi (grands-mères), composé des premières disciples de Lekhraj. Ce dernier fait toujours l'objet d'une intense dévotion, à travers un phénomène de transe institutionnalisée : les BK vénèrent une entité immatérielle, nommée Shiv Baba, amalgame composé de Dieu et du fondateur, s'exprimant annuellement à travers l'une des dadi octogénaires dirigeantes. Cette valorisation du paranormal n'empêche ni la mise en avant d'une vocation scientifique, ni la multiplication de séminaires de self et stress management à l'intention des élites de tout secteur social (industrie, finance, presse, sport, art, etc.). Prosélyte et ambitieuse, la BKWSU multiplie conférences et colloques, et mobilise les technologies de médiatisation les plus abouties : infrastructures et logistiques ultramodernes, supports audiovisuels sophistiqués, réseaux de communication étendus, etc. Elles relient le Mont Abu aux BK du monde, estimés à un demi-million d'individus par leurs responsables. Tous, les membres de la diaspora en priorité, sont invités à participer à la construction de l'Inde triomphante de demain, exemplaire en sa spiritualité, sa droiture et son homogénéité. La BKWSU partage plus d'une idée avec les mouvements nationalistes hindous les plus vindicatifs.

\section{Le benjamin}

The Art of Living, fondé en 1982 dans la "Silicone Valley " indienne de Bangalore, affiche sa tolérance et valide toutes les religions. En tant que « fondation pour le développement personnel », elle s'autorise les mélanges les plus

10. Ensemble des prescriptions (dharma) auxquelles se soumet l'hindou selon son groupe d'appartenance social (varna) et son âge (ashrama). 
audacieux entre les techniques hindoues de dépassement de soi et les savoir-faire occidentaux, aboutissant à un produit fortement teinté de New Age. Elle dépend d'un gourou flottant, Shri Shri Ravi Shankar, qui ne confère nulle initiation privée, mais transmet à qui veut bien l'acheter une technique respiratoire curative, le sudarshan kriya, enseignée lors de stages intensifs. Sa renommée et ses actions, étendues à l'ensemble du territoire indien, donnent à son groupe une envergure internationale.

Shri Shri Ravi Shankar est né en 1956 dans une famille brahmane du Tamil Nadu. Il reçut une solide formation religieuse, notamment sous l'égide du professeur de sanskrit de Gandhi, Pandit Sudakar Chatunvedi, avant de poursuivre sa scolarité au lycée Saint-Joseph de Bangalore. Il s’y révéla particulièrement doué pour les mathématiques et la physique, ce qui contribue, aujourd'hui, à donner de lui l'image d'un scientifique à la tête d'une institution dédiée à la recherche. Cependant, pénétré de spiritualité, il préféra à la vie mondaine l'ashram de Maharishi, père de la "Méditation Transcendantale » (MT) et figure clef de la diffusion du yoga en Occident dans les années $1970^{11}$. Après plusieurs années passées auprès du maître, Shri Shri s'en sépara et connut une expérience décisive en 1982, lorsqu'il obtint la révélation du sudarshan kriyā. C'est afin de le transmettre au monde qu'il fonda The Art of Living, implanté aujourd'hui dans cent quarante pays. Depuis, il ne mentionne qu'exceptionnellement Maharishi, et se réfère ponctuellement au maître de ce dernier, Swami Brahmananda Saraswati. Bien qu'héritier d'une brève lignée, il ne s’inscrit pas vraiment dans sa continuité et ne doit son succès qu'à ses talents.

Au-delà de sa connaissance, réputée parfaite, des textes sanskrits anciens, S.S. Ravi Shankar délivre un message syncrétique : l'universalisme spirituel, à travers la dévotion envers le divin au sens large " God is one for everyone ", et, plutôt que de vanter telle ou telle voie ascétique ou cultuelle, invite à vénérer et à cultiver la joie à travers le rire, la danse et le chant. C'est ainsi que chaque antenne d'AoL organise à la fois des satsang, assemblées gratuites et ouvertes à tous, strictement consacrées au chant dévotionnel et inscrites dans l'orthopraxie hindoue ordinaire, et des stages de développement personnel, nourris, eux, des techniques anglo-saxonnes de rapport à soi les plus actuelles. AoL ne possède ni temple ni dogme, et n'admet que l'empirisme : toutes les techniques enseignées visent le mieux-être, physique comme psychique. Le postulat est que tout individu ayant stabilisé son épanouissement personnel se trouve en mesure de favoriser celui des autres à travers l'aide humanitaire. En résumé, S.S. Ravi Shankar incarne un gourou à la fois classique et original.

11. Maharishi fait figure de pionnier dans la tentative de hisser la pratique spirituelle au rang de discipline rationnelle. Refusant d'en parler en termes de nouvelle pratique religieuse, il lui attribuait des fondements savants (la connaissance du système nerveux central) et des effets curatifs (la santé mentale et physique), pour l'envisager comme une technique scientifique révolutionnaire, qu'il défendait lors de ses tournées à travers le sous-continent. 
Classique, en premier lieu parce que la formidable attraction qu'il suscite s'exprime à travers les concepts et les pratiques de la dévotion (bhakti) traditionnelle ${ }^{12}$; ensuite, parce qu'il perpétue la tradition du yoga ; enfin, parce qu'il ne fait figure ni de prophète, ni de réformateur. L'hindouisme présente, selon lui, toutes les qualités requises pour articuler une pensée et une pratique universelles, et ne doit pas plus que les autres religions être dévalorisé ou modifié. Original, parce qu'il n'impose rien, et exige peu : son autorité repose sur ses pouvoirs de guérison, assimilés par ses disciples à la magie et au surnaturel. Il réalise donc une symbiose attractive entre procédés curatifs et techniques de salut. Enfin, il multiplie les missions humanitaires et écologiques qui valent à AoL d'être, elle aussi, une ONG.

S.S. Ravi Shankar ne s'attache pas seulement au sort des déshérités, mais se consacre intensément aux pathologies "sociales ", dont souffrent les individus trop insérés dans les rouages de la société ! La charité dispensée diffère alors des offres classiques de soins, de nourriture et d'alphabétisation, généralement proposées par les organisations charitables. Le secours apporté par AoL aux "repus " ${ }^{13}$ mal dans leur peau les initie au stress management et à la catharsis. On ne s'étonnera donc pas que le siège mondial de AoL se situe à Bangalore : depuis l'ouverture indienne au libéralisme économique, au début des années 1990, la capitale du Karnataka incarne "l'Inde mondialisante ", tant elle concentre de sociétés privées performantes et de brillants « cerveaux » rompus aux techniques IT, c'est-à-dire les technologies de l'information. Cinquième ville de l'Inde, Bangalore promet à une population jeune et ambitieuse les hauts niveaux de formation requis pour être acteurs de la mondialisation et non simples spectateurs passifs. AoL y tient ses quartiers, puisque ses séminaires les plus lucratifs s'adressent en priorité aux étudiants anxieux de réussir dans la vie et aux affairistes ayant gravi les échelons du succès au prix d'un intense surmenage. Les programmes de ses interventions à travers le monde introduisent le gourou d'AoL comme "Éminent conférencier et Humaniste de renommée mondiale » ${ }^{14}$. Petit et gracieux, invariablement drapé dans des voiles blancs flottant autour de lui, Shri Shri ne cesse d'affirmer qu'il cultive l'enfant demeuré en lui, en dépit de ses presque cinquante ans. Ses cheveux et sa barbe rappellent Maharishi, mais il se plaît à se comparer au dieu Krishna, rapprochement que ses disciples approuvent en lui attribuant la malice, la spontanéité et la bonté caractéristiques de l'avatara

12. Principalement le darshan (échange de regard entre le fidèle et l'objet de sa dévotion), le culte et le service, du gourou (guru puja, guru sevā).

13. Selon l'expression de Danièle Hervieu-LÉger, "Les tendances du religieux en Europe », in Croyances religieuses, morales et éthiques dans le processus de construction européenne, Paris, La Documentation Française, 2002, p. 16.

14. Il a participé, en tant qu'intervenant de marque, à de nombreux symposium internationaux, comme le Forum économique mondial de Davos, le Sommet du Millénaire pour la Paix à l'ONU à New York, la Conférence interreligieuse des représentants des religions du monde à Séville, ou encore la Conférence mondiale pour la gestion de l'environnement. 
de Vishnou. Son allure avenante et son tempérament expansif désignent Shri Shri comme l'incarnation d'une nouvelle sorte de sainteté, adaptée au monde moderne, et dont l'originalité réside en l'exceptionnelle attention qu'il accorde aux domaines sociaux et sanitaires.

En conclusion, chacun des trois mouvements dépend d'une autorité bienfaitrice de l'humanité, puisant dans son projet altruiste et englobant de quoi justifier son ascendant. Au charisme passif du gourou traditionnel se substitue le rayonnement de l'action de grâce. Loin de se contenter de transmettre, le gourou flottant regarde vers l'avenir et agit en faveur du changement. Porteur d'espoir, il laisse entrevoir, enfin, le possible réveil d'une fierté ethnique piétinée par l'histoire. Bien que l'on assiste à une transformation sensible des figures d'autorité, une même emphase sur l'importance de contenter le maître se retrouve dans le BAPS, AoL, et la BKWSU (en dépit du fait, dans ce dernier cas, qu'il soit décédé). À la question "pourquoi faites-vous ce que vous faites? ", les membres de chacune des trois communautés répondent volontiers : "pour lui». «Lui » (Pramukh Swami, Dada Lekhraj, Shri Shri Ravi Shankar), assure à la fois l'unité du groupe et la cohésion mentale du disciple. Les qualités conférées au gourou induisent que ses fonctions débordent le seul cadre des attributions religieuses et de leurs applications locales. Universel, le maître est une figure clef du phénomène de transnationalité. L'instrumentalisation de bhakti et sevā appuie l'ensemble de ces dynamiques.

\section{La bhakti, vecteur transnational de solidarité civile}

Le BAPS, la BKWSU et AoL sont originaires de régions historiques propices à l'épanouissement de la dévotion. Berceau du dernier, le Karnataka fut un haut lieu de la bhakti vishnouite "émotionnelle " ${ }^{15}$, alors que les deux premiers ont grandi dans l'Inde du nord-ouest imprégnée d'un krishnaïsme amoureux et lyrique, - dans laquelle s'est déroulée l'essentiel de notre enquête.

Soucieux de mobiliser une version contemporaine de la dévotion, le BAPS, la BKWSU et AoL jouent de la malléabilité même de la notion de renoncement. L'hindouisme, en effet, l'entend sur au moins trois modes : le premier concerne sa forme radicale, "orthodoxe" (samnyāsa), telle que l'a définie le système brahmanique du varna-aśrama-dharmạ, et équivaut à une mise à l'écart du monde en conclusion d'une vie de devoir, initialement réservée aux brahmanes; le deuxième, plus élargi, privilégie au contraire son application intramondaine, «adoucie » parce qu'intériorisée, propre à la voie de la dévotion pieuse décrite par le texte majeur de la Bhagavad Gita; le troisième embrasse les infinies

15. Depuis l'action prosélyte, dès le vII ${ }^{\mathrm{e}}$ siècle, des Alvar vishnouites, la bhakti du sud de l'Inde valide la totalité de l'expérience humaine, en exprimant l'ouverture sensuelle du dévot à Dieu, accessible en partie au travers des sens. 
déclinaisons des nombreux courants relevant de la bhakti populaire, accessible à tous, dont les sampradāya vishnouites sont l'illustration.

Ces institutions s'emploient surtout à établir une éthique, confondue avec une pratique de la dévotion inspirée de la Bhagavad Gita, ouvrage fondateur de la bhakti. Livre six de la célèbre épopée du Mahabharata, le "Chant du Bienheureux Seigneur " met en scène le dieu Krishna, venu discourir sur les bienfaits de la discipline dévotionnelle, afin de convaincre le héros guerrier Arjuna que tout acte accompli en adéquation avec son dharma personnel, même le plus meurtrier, vaut salut si la piété l'accompagne. Il suffit, explique-t-il, d'agir impersonnellement, en dédiant l'action au divin jusqu'à la vivre comme une forme de sacrifice intériorisé, c'est-à-dire en renonçant à ses fruits. Dès lors, renoncer ne se confond plus avec le non-agir, puisque la bhakti en propose une version dé-ritualisée, réhabilitant l'acte ordinaire de manière à offrir à tout individu la possibilité de se réaliser à travers lui, en le créditant des mêmes pouvoirs de libération que la gnose brahmanique ou le rituel. Elle n'exige nulle autre connaissance que celle, intime et intuitive, du divin, et ne requiert l'intervention d'aucun spécialiste du sacré.

Telle est la révolution opérée par la bhakti: une nouvelle chance de libération, étendue à tous, basses castes et femmes incluses, ce qui en fera la voie de salut la plus populaire dès le tournant de notre ère et la plus potentiellement transgressive au regard du dharma. Parce qu'elle consiste à cultiver en soi l'amour et le souvenir de son dieu d'élection, la bhakti demande au dévot de déployer ses potentialités d'ordre sentimental et émotionnel, d'où sa déclinaison en une infinité de "vécus " et d'expressions possibles, - ce qui explique qu'elle soit responsable de la floraison des sampradaya depuis la seconde moitié du premier millénaire de l'ère chrétienne. Ceci se justifie d'autant plus que la bhakti assure et entretient l'autorité du gourou : d'abord parce qu'elle s'adresse au dieu et au maître en les confondant (la plupart des communautés regardent leur gourou comme une incarnation divine) ; ensuite parce qu'elle s'exprime à travers le service divin (sevā), accompli par le fidèle pour le bien-être de son dieu - et, corollairement, de son gourou.

\section{L'ONG-isme ou la dévotion civile}

Si le BAPS, la BKWSU et AoL récoltent aujourd'hui les fruits d'une transnationalité " galopante ", c'est qu'ils ont su s'approprier des dynamiques transversales aptes à les inscrire dans la durée : ils ont connu une deuxième naissance à la faveur de la mouvance New Age de la décennie 1970, et une troisième grâce à la vague humanitaire des années 1980. Le choix d'une vocation spirituelle « humanisante » recèle davantage qu'un appétit personnel de croissance ; chacun, de manière plus ou moins prononcée, arbore une fierté nationaliste l'amenant à défendre une certaine indianité. L'Inde à laquelle le BAPS, la BKWSU et AoL se dévouent est somme toute une Inde de la performance, dont ils s'accordent à 
louer les virtuosités, tant technologiques que spirituels. En regard des puissances occidentales déclinantes, sa progression économique va à une telle cadence et couvre tant d'espace que le sous-continent peut envisager d'inverser les rôles, quitte à remobiliser un certain esprit néo-hindouiste : l'Inde hériterait de la mission civilisatrice autrefois apanage de l'Occident. Les groupes qui nous occupent y travaillent en conjuguant habilement deux contrastes, l'universalisme et le particularisme, habileté qui garantit leur succès auprès d'un public indien partagé entre l'un et l'autre. Tandis que les valeurs de la modernité occidentale prônent un égalitarisme de nature, le code social hindou se construit selon une biérarchie de nature. Face à cette antinomie, le BAPS, la BWKSU et AoL occupent une position d'entre-deux : ils ne remettent pas en cause la disparité innée des êtres, mais ne s'en contentent pas non plus, désireux de jouer le jeu du progressisme. Leur démarche consiste à substituer à l'égalitarisme par l'être (acquis) un égalitarisme par le faire (conquis).

À travers le paradigme que j'ai choisi d'appeler "ONG-isme », le gourou s'octroie la dimension du Sauveur. S'il y ajoute celle d'agitateur public, il peut toucher au nationalisme, d'autant que le fondamentalisme hindou aime à faire de ses militants des modèles ascétiques ${ }^{16}$. Dans l'un et l'autre cas, le charisme légitime le changement et le gourou jouit d'une tribune. D'aucuns jouent de l'élasticité de bhakti et sevā pour l'étirer jusqu'au politique, en arguant que l'Inde est avant tout une entité religieuse, dont la grandeur spirituelle justifie, d'une part, que tout résident s'y dévoue comme à un maître ou un dieu, et de l'autre, que le monde entier la reconnaisse comme telle. L'agressivité nationaliste complète alors l'argument universaliste, renforçant l'alliance paradoxale de l'ancrage local et de la déterritorialisation cultivée par les organisations observées.

Trois champs d'application sensibles permettent au BAPS, à la BKWSU et à AoL d'exprimer avec succès leur investissement humanitaire :

- L'aide d'urgence : les catastrophes naturelles que l'Inde subit régulièrement en font un terrain d'exercice privilégié. Le tremblement de terre survenu dans la région du Kuch (Gujarat) en janvier 2001 offre une bonne illustration de l'attitude des trois ordres face au drame. À en croire la BKWSU, les bâtiments de la région sinistrée abritant des centres de raja yoga furent parmi les seuls à rester debout : dès les premières secousses, les BK s'y assirent en méditation, déterminés à ne pas céder à la panique et à se tenir prêts, en toute quiétude, pour la destruction. Leur rôle était défini par avance : montrer l'exemple d'une stabilité à toute épreuve. Les santos du BAPS partirent en convoi d'ambulances pour organiser des distributions de nourriture et de biens de premier secours, avant d'ébaucher les premiers plans architecturaux de reconstruction. Les bénévoles d'AoL, après avoir aidé à la sécurité des corps, s'occupèrent des âmes : en « cellule psychologique de soutien ", ils proposèrent d'effacer les blessures morales traumatiques par des séances de sudarshan kriya.

16. Christophe Jaffrelot, Les nationalistes hindous, Paris, Presses de la FNSP, 1993, p. 52. 
- L'éducation : les trois mouvements défendent que l'âge d'or marqua l'ère de la science infuse, tandis que l'âge de fer vit sous le règne de l'ignorance. Maîtres du savoir, les hommes de l'antique Bharat détenaient tous les pouvoirs : esclaves des forces obscurantistes, ceux de l'Inde moderne ne se possèdent même pas euxmêmes. Le BAPS et la BKWSU expriment nettement leur militantisme en faveur d'une réinsertion du religieux dans les établissements scolaires: l'éducation représente pour les classes moyennes indiennes un terrain sensible, l'opinion tenant en basse estime les écoles publiques dont elle juge le niveau médiocre et les équipements insuffisants. On s'affirme convaincu qu'il est crucial d'assurer la compétitivité des enfants sur l'échiquier économique international en les confiant aux soins des florissantes écoles privées anglophones. Ces établissements privés, souvent d'obédience chrétienne, se monnaient chèrement. Les trois institutions multiplient la création d'établissements scolaires ultra-modernes où se transmettent les fondements principaux de la culture hindoue.

- L'écologie : les préoccupations humanistes et humanitaires accueillent depuis peu un paradigme nouveau : l'écologie. Est-ce parce que l'on conçoit l'équilibre du monde en un tout autre rapport de forces que l'on se montre si peu soucieux, en Inde, de l'environnement ? Contre cette négligence, la BKWSU et AoL agissent en pionnières en expérimentant les énergies douces, en multipliant les actions d'épuration, de recyclage et de reboisement. Leur objectif est l'assainissement, de l'individu dans un premier temps, de l'environnement dans un second. S'il est un réenchantement possible, il ne se conçoit pas comme la re-magification ou le repeuplement de l'environnement, mais comme sa transformation en un monde meilleur... et propre.

Ces trois domaines d'intervention offrent des cadres propices au développement et à l'application du concept de sevā, entendu comme service désintéressé et méritoire rendu par l'individu dépourvu d'ego au bien de l'humanité. Le statut d'ONG constitue l'une des spécificités les plus irréductibles de ces trois institutions. Il ne représente pas seulement l'un de leurs principaux atouts auprès du public, mais renseigne, aussi, sur l'actuelle propension de l'hindouisme à s'expatrier en s'universalisant. Au regard des ancrages de l'idéologie humanitaire au sein de la pensée chrétienne, que des Églises détiennent un statut et des facilités d'ONG ne doit pas nous étonner, même si la systématisation du recours à cette stratégie (de recrutement, de légitimité, de pouvoir, de richesse) nous laisse songeur. Nettement plus surprenante s'avère, au contraire, l'épanouissement de ce type d'entité en Inde, puisque les valeurs humanistes de charité et d'assistance sont rarement mises en avant dans la culture d'inspiration brahmanique. Max Weber, déjà, notait que celle-ci ignorant l'absence de droit naturel des hommes et des choses, « il n'existait en effet aucune sorte d'égalité naturelle de l'humanité devant une quelconque instance, et surtout pas devant un quelconque "Dieu" supramondain ${ }^{17}$. Rappelons brièvement la contextualité propre à la culture hindoue. La

17. Max Weber, Hindouisme et bouddhisme, Paris, Champs Flammarion, 2003, p. 258. 
loi du karma, génératrice de rétribution personnelle, a freiné l'établissement de droits et de devoirs communs. En conséquence, "l'émergence de spéculations de critique sociale et d'abstractions rationalistes, au sens de droit naturel, était entièrement et définitivement exclue, de même que la constitution de quelconques "droits de l'homme" ". La pensée hindoue, explique Weber, exempte de "péché en soi ", ne compte que les seules entorses rituelles au dharma concret ; inégaux, les hommes ne se voyaient pas condamner par un « mal radical ", mais pouvaient s'élever par la voie de la renaissance. Centré sur l'épanouissement de la personne, et fortement teinté d'individualisme, l'humanisme demeure une valeur plutôt exogène sur la terre indienne, en laquelle l'individualité est une catégorie plus fonctionnelle que substantielle.

Le BAPS, la BKWSU et AoL luttent pour démentir cet état de fait, au moyen de la dévotion et de la sevā. À l'égal de la solidarité, la bhakti traverse les frontières. Son essence universaliste en fait une force transnationale, pour peu qu'elle serve la «bonne cause ", de sorte que dévotion religieuse et dévouement humanitaire finissent par ne faire qu'un. Mark Juergensmeyer tire à ce sujet d'édifiantes conclusions de son observation des Radhasoami, secte utopique née en 1860 dans la communauté marchande d'Agra ${ }^{18}$. Marginal, le groupe juge idéale l'existence hors monde et hors ascétisme à la fois, et prêche le détachement et le service social, même s'ils semblent à première vue obéir à des motivations antinomiques. Il revient à la sevā de résoudre la contradiction, et d'assurer la médiation entre ces deux extrêmes que sont le renoncement et la vie mondaine : elle offre à l'individu l'opportunité de s'effacer derrière le service des autres, et de répondre aux besoins du monde sans se soucier des siens propres. La sevā permet de tisser un lien subtil entre des vocations mêlées, à la fois individualiste et holiste. Dans ce contexte, elle est fondatrice et synonyme d'une nouvelle éthique assurant le passage entre le spirituel supramondain et la morale appliquée ici-bas.

En résumé, bhakti et sevā, de leur statut initial de piliers de l'orthopraxie, deviennent arguments idéologiques. Leur instrumentalisation sous-tend un glissement progressif de l'attitude rituelle individuelle à la valeur morale collective, puis de la morale à un processus d'idéologisation (ils servent une construction intellectuelle justifiant des intérêts). Ces trois institutions s'entendent à faire glisser des attitudes sacrées ancestrales, du champ religieux vers le champ de l'éthique et du progressisme social. La dévotion ne se vit plus dans un esprit d'intimité et de libre arbitre. D'émotion, elle devient discipline, attitude non seulement morale mais moralisatrice. Le service, traditionnel engagement à la divinité et au gourou, rejoint progressivement le devoir humanitaire. Bhakti et sevā relèvent de l'action militante, leur vocation est uniformisante, leur redéfinition vise à faire d'eux des universaux.

18. Mark JuergensmeYer, Radhasoami Reality. The Logic of a modern Faith, Princeton University Press, 1991. 


\section{De la bhakti au sanatana dharma fédérateur}

Les trois institutions ont à leur tête des leaders fortement mobilisés pour la prétention à la supériorité indienne de l'Inde. Elles participent, de façon plus ou moins prononcée, à la fiction idéologique du «mythe des origines ». Le BAPS néglige tout pluralisme religieux, en confondant volontairement culture indienne et culture hindoue : il ne fait aucune allusion à la composante islamique, pourtant hautement constitutive de l'histoire et de la civilisation du sous-continent. On doit d'autant moins sous-estimer une telle " omission » que les récurrentes violences communalistes entre hindous et musulmans s'avèrent particulièrement soutenues dans le Gujarat, devenu dans les années 1990 le bastion des nationalistes hindous. Les massacres perpétrés au printemps 2002, à Ahmedabad et dans plusieurs villes et villages gujarati, ont atteint un paroxysme. À leur suite, les musulmans se sont retranchés dans des ghettos que l'institution n'a pas jugé nécessaire, cette fois, de visiter. On comprendra, dans ces conditions, que le message du BAPS ne peut s'entendre comme neutre.

La BKWSU, de son côté, diffuse un message ouvertement offensif. Son biographe, Jagdish Chander tient des propos agressifs et contestables dans son ouvrage intitulé Eternal Drama, où il confond l'Inde avec le berceau de l'humanité ${ }^{19}$, soutient que le Veda démontre l'antique supériorité du peuple aryen, premier groupe autochtone indien (sic), et utilise cet argument pour défendre une redoutable fierté ethnique. Il prophétise avec arrogance la prochaine hégémonie indienne sur le monde. L'affirmation de la supériorité indienne s'appuie sur l'idée de sanatana dharma, désignant dans les textes brahmaniques la religion parfaite, éternelle et immuable de l'humanité. Pour le BAPS, la BKWSU et AoL, comme pour les partis nationalistes hindous membres du Sangh Parivar (notamment le RSS et la VHP), l'Inde en serait la matrice et recèlerait toutes les qualités pourvu qu'elle « redevienne » (sic) le siège d'une culture unifiée, offrant à l'humanité défaillante le seul système socioreligieux viable. Récurrente, l'expression sanatana dharma se voit instrumentalisée au point de devenir un véritable "sésame ". Littéralement, le mot dharma évoque la loi religieuse et cosmologique, ainsi que l'acte accompli en accord avec cette loi, soit un ordre supra-individuel. Teinté d'humanisme, d'égalitarisme et d'universalisme, le dharma se mue en la "nouvelle ancienne religion » appelée à racheter l'humanité sitôt l'Inde réformée. Partie prenante de cette hypertrophie, les néo-hindous prêchaient toutefois une religion qui, bien qu'universelle, devait s'exprimer à travers des formes locales dignes de respect.

C'est sans doute pour insister sur son universalité potentielle et le débarrasser d'une contextualité sociale jugée trop étroite que le dharma fait peu à peu place au sanatana dharma. Initialement, l'expression fait référence à la norme brahmanique élevée au rang d'absolue Vérité, et affranchie de toute limite spatio-temporelle. Elle en arrive à désigner un ensemble de fondamentaux contribuant à définir une prétendue orthodoxie hindoue : dès sa fondation en 1875, la Mission Ramakrishna

19. Jagdish Chander, The Eternal Drama. Part 2, Mont Abu, Prajapita BKIVV, 1985. 
l'entend comme l'essence même de la dévotion, caractérisée par la sincérité, le sérieux, la concentration, le renoncement à tout attachement ${ }^{20}$; Gandhi, pour sa part, la sollicite en 1921 pour défendre sa version de "l'hindouisme immuable ", qui induirait selon lui la foi dans les Écritures, la transmigration et le varnaashrama-dharma, ainsi que la protection de la vache et le culte des images ${ }^{21}$. Par extension, le sanatana dharma désigne l'hindouisme en une dimension universelle, sous-entendant un «Ordre éternel » contre lequel on ne peut rien. La formule offre l'avantage, d'une part, de se relier à un fond culturel commun sans pour autant se dire hindouiste sectaire, et de l'autre, d'affirmer que l'Inde a engendré une spiritualité homogène, inaltérable et incontournable. Les néo-hindous les plus radicaux, les nationalistes hindous, ou encore les Brahma Kumaris et autres courants à tendance New Age qui en font grand usage, la traduisent en termes de "religion authentique universelle ». Le sanatana dharma est, pour ces artisans d'une certaine modernité indienne, un idéal auquel il faut tendre, qui permettrait à l'homme de renouer avec sa véritable nature - celle qui s'est perdue dans la nuit des temps mais qui se tient toujours à portée de main, prête à renaître pour peu qu'on lui consacre les efforts adéquats. On peut avancer que la plupart des mouvements de renouveau indiens usent de l'expression ${ }^{22}$. Les groupes qui défendent leur condition privilégiée d'ONG, ont tout intérêt à entretenir l'apologie d'une Vérité universelle, présente de toute éternité au point d'être indissociable de l'humanité.

\section{Au cour de la cible}

Ces maîtres à penser charismatiques provoquent autour d'eux zèle et empressement en proposant un produit conforme aux attentes d'individus à la fois inscrits dans une continuité (pérennisant une attitude dévotionnelle envers le gourou largement éprouvée par leur tradition), et prêts à s'affranchir des legs les moins appropriés à leur mode de vie (acteurs d'une dynamique propice à la mise en libre-service du religieux). Leur aptitude au "bricolage " séduit une population dont nous pouvons esquisser le profil au moyen de quelques traits.

Qui fréquente la société indienne depuis deux décennies observe que la libéralisation économique a fait naître une nouvelle classe sociale, urbaine et entreprenante, à la fois réceptive aux concepts de la modernité occidentale et soucieuse de protéger un particularisme identitaire, souvent déterminé par l'appartenance religieuse. Ses référents culturels, son niveau de vie, l'inscrivent dans ce que l'on

20. R.W. NEufELDT, "The response of the Ramakrishna Mission », in Harold G. Coward, ed., Modern Indian Responses to Religious Pluralism, New York, State University of New York Press, 1987, p. 73. p. 230 .

21. T.N. Madan, Modern Myths. Locked Minds, Delhi, Oxford University Press, 1997,

22. R.D. BAIRD, "The response of Swami Bhaktivedanta ", in Harold G. CowARD, éd., Modern Indian Responses to Religious Pluralism, op. cit., pp. 111-112. 
a tendance à nommer classe moyenne, catégorie dans laquelle elle-même se situe volontiers. Le National Council of Applied Economic Research estime que rentrent dans cette catégorie tous ceux bénéficiant d'un revenu annuel supérieur à 3400 euros $^{23}$, proche du standard occidental. Leur population aurait quadruplé en dix ans, représenterait début 2006 un peu plus de $10 \%$ du milliard d'Indiens et s'accroîtrait chaque année de quelque trente millions d'individus ${ }^{24}$.

L'idéal culturel nommé middle class que l'Inde a progressivement construit assure aujourd'hui la popularité d'une catégorie qui mobilise les représentations collectives bien au-delà de ses réalités empiriques. Même si l'expression de «classes consuméristes ", traduction quelque peu pesante de l'heureuse formule anglaise consuming classes, lui semble préférable, une précaution s'impose étant donné que la jouissance de biens de consommation ne suffit certainement pas à la définir. Telle qu'elle apparaît dans les discours ambiants, elle ne se distingue pas seulement par un accès facilité à la consommation, mais hiérarchise aussi ses membres en hautement ou médiocrement educated. Si l'on voulait risquer une généralisation, en dépit de gênants raccourcis, on pourrait avancer que les acteurs de la classe moyenne indienne : ont été scolarisés dans la durée, et éventuellement diplômés ; sont tous plus ou moins anglophones ; exercent une activité professionnelle et bénéficient de revenus qui, sans être très élevés, ont l'avantage d'être réguliers ; maintiennent un contact étroit avec les médias ; ont accès aux loisirs.

Outre leur inscription dans ces classes, le BAPS, la BKWSU et AoL reçoivent une part importante de leurs subsides de la diaspora indienne, estimée à une vingtaine de millions d'individus. Notable sur le continent africain depuis deux cents ans, elle s'est intensivement transférée vers l'Ouest à la faveur du dynamisme économique des années 1960 et de la levée des restrictions américaines à l'immigration. Son succès contribua amplement à entretenir en Inde un « rêve américain ", et à cristalliser une vision idéale de la classe moyenne entreprenante et épanouie ${ }^{25}$. Si la première génération d'immigrants a globalement vu le jour et grandi en Inde, la seconde, elle, est moins intime avec sa terre d'origine. Les nostalgies entretenues par l'une et l'autre diffèrent ${ }^{26}$.

23. Soit environ 15000 roupies par mois. Les «cols blancs " peuvent atteindre ce niveau, tandis que le salaire mensuel moyen d'un enseignant scolaire s'élève à 3000 roupies, et qu'un ouvrier agricole gagne, au mieux, à peine la moitié de cette somme.

24. Sources : hebdomadaire Challenges, $\mathrm{n}^{\circ}$ 24, 23 février 2006, p. 54 ; mensuel Capital, $\mathrm{n}^{\circ} 174$, mars 2006, p. 54.

25. Ce dernier point s'avère d'autant plus crucial que les Indiens implantés aux États-Unis y forment l'une des minorités ethniques les mieux éduquées, les plus réalisées professionnellement et prospères.

26. Arvind Rajagopal considère ladite nostalgie comme indissociable du statut liminal de la première génération, et qu'elle s'exprime à travers son application assidue au rituel. Les enfants nés à l'étranger, en revanche, mieux intégrés, se montrent plus sensibles à une Inde mythifiée qu'à l'Inde-territoire - qu'ils auraient même plutôt tendance à réprouver pour ses archaïsmes et ses inconforts (Politics after Television. Hindu Nationalism and the Reshaping of the Public in India, Cambridge University Press, 2001). 
La diaspora, en particulier aux États-Unis, constitue une sorte d'apothéose de la classe moyenne supérieure indienne, l'exemple à suivre d'une success story : en affichant le mérite et le potentiel de l'indianité, elle démontre que celle-ci recèle toutes les qualités autorisant un Indien à réussir mieux que quiconque, en dépit des conditions défavorables offertes par le sous-continent. L'attachement à cette indianité passe manifestement par le religieux : Raymond Brady Williams, spécialiste du BAPS, remarque que les Gujarati installés en Grande Bretagne tendent à se dire plus religieux une fois installés dans leur pays d'accueil qu'auparavant en leur patrie ${ }^{27}$. Manifestement, la perception d'elle-même autant que les anxiétés ${ }^{28}$ de cette population croisent favorablement la démarche nationaliste de la VHP, qui consiste à appuyer la valeur de l'indianité sur l'hindutva. Les populations diasporiques d'ingénieurs et de managers, surtout, se montrent fort sensibles aux sollicitations politiques de "l'Association Hindoue Universelle » (à l'inverse de l'Inde, où l'organisation connaît moins de succès auprès d'elles qu'auprès des lower-middle class ${ }^{29}$ ). D'une manière comparable aux communautés que nous avons étudiées, elle se développe en s'investissant intensivement dans les fondations sociales et charitables - hôpitaux, écoles, foyers, etc. La récurrence de ces similitudes a quelque chose à nous dire de la personnalité diasporique, et leur analyse in situ stimulerait sans doute de nouveaux débats autour de l'acculturation et de l'interaction. Relais économique, la diaspora établit aussi des ponts idéologiques - les médias ne l'ont que trop mentionné à propos du 11 septembre et des appuis britanniques de l'organisation terroriste Al Qaida. On peut supposer que les compromis qu'elle doit concéder compromettent parfois le confort de son intégration, et que c'est cet inconfort même qui justifie son attirance pour des mouvements au déterminisme rassurant. Mais on peut aussi envisager que son rêve de toute-puissance de l'Inde résulte moins de sa fragilité que de sa confiance en elle. Le BAPS, la BKWSU et AoL jouent de fait sur les deux tableaux, en avançant un discours à la fois alarmiste sur l'instabilité affectant les populations hindoues immigrées, et triomphal (parfois jusqu'à l'arrogance) sur la force conquérante dont elles menacent les sociétés au sein desquelles elles évoluent.

\section{Conclusion}

Le BAPS, la BKWSU et AoL présentent les qualités de groupes structurés et visibles, favorables au leadership charismatique et d'envergure internationale, que l'on qualifie depuis quelque trente ans en Occident de « Nouveaux Mouvements

27. Raymond B. Williams, An Introduction to Swaminaryan Hinduism, Cambridge University Press, 2001, p. 198.

28. Arvind Rajagopal avance que la première génération d'immigrés connaît de violentes remises en question lorsque ses enfants rejettent l'autorité de la tradition pour mieux se fondre dans la culture de leur terre d'adoption (op. cit., p. 264).

29. Arvind RajAgOPal, op. cit., p. 267. Chris Fuller rapporte le même constat (communication orale, EHESS, février 2006). 
Religieux » (NMR). Ils se présentent à la fois comme des mouvements-refuges, des groupes de réalisation de soi et des tentatives de revivalisme religieux. Nous ne devons pas pour autant oublier que leurs conditions d'émergence et celles des NMR occidentaux diffèrent. De notre côté du globe, les sociologues des religions débattent pour décider si New Age et NMR signalent le renforcement de la sécularisation ou, au contraire, de la religion. La nécessité de redéfinir la notion de laïcité s'impose à eux, dès lors que la crise des institutions de régulation du croire ne va pas systématiquement de pair avec la crise du religieux. L'effondrement institutionnel occidental a-t-il son pendant indien ? Une étude comparative des modèles laïcs respectifs serait riche d'enseignements.

Malgré la laïcisation entreprise par Nehru après l'Indépendance, la difficulté même de circonscrire en Inde une religion majoritaire, l'absence d'institution ecclésiale centralisatrice, et enfin l'étroite imbrication entre le sacré et le profane dans l'espace social, écartent toute idée de séparation radicale entre l'État et le religieux. La Constitution pose que le sécularisme indien consiste d'abord à répondre au pluralisme religieux propre au sous-continent, et à protéger les droits des minorités en garantissant en premier lieu la neutralité étatique.

L'absence de toute théologie consensuelle rend inapplicable à l'Inde le modèle américano-européen dont on entend s'inspirer, modèle qui tient davantage de la liberté de conscience que de la liberté religieuse. C'est pourquoi, comme le souligne très justement Marc Galanter, la plupart des indiens amenés à réfléchir à la question n'assimilent pas le secularism au "non religieux » mais au «non sectaire » ${ }^{30}$, c'est-à-dire à l'homogénéisation des modes pluriels de croyances et d'existences (qui sont jugés responsables, en cas de dérégulation, du communalisme). Loin d'appeler systématiquement à la soumission à une religion déterminée - à commencer par la plus pratiquée : l'hindouisme -, ils envisagent généralement un consensus autour d'une foi universelle en un dieu unique qui, se plaisent-ils à affirmer, " est ultimement le même pour tous ". On conviendra que les organisations présentées ci-dessus font écho à cette aspiration au consensus, en recourant notamment à la notion de sanatana dharma.

Le modèle des gourous flottants nous aide à comprendre comment les tensions jouant entre formes universalisantes et particularisantes s'intensifient avec la mondialisation. C'est pourquoi le terme de "glocalisation » nous semble particulièrement propice à préciser le paradoxe d'un phénomène socioreligieux suscitant autant de dé- que de re-territorialisation. Chantal Saint-Blancat explique que la globalisation introduit une hybridité multiple, qui ne correspond pas à une simple adaptation mais à un processus complexe où, au contact de systèmes culturels différenciés, des modèles universels, notamment les religieux, se transforment et

30. Marc Galanter, "Secularism East and West ", in Rajeev BHARGava, éd., Secularism and its Critics, Oxford University Press, 1998, p. 234-267. Ce point mérite d'être souligné car les nombreux entretiens menés dans le cadre de notre enquête le confirment amplement. 
acquièrent leur propre autonomie ${ }^{31}$. De fait, la religion exprime ces tensions avec une remarquable acuité ${ }^{32}$. La réalité locale qui nous retient ici s'appuie sur la vitalité des réseaux transnationaux à travers laquelle la globalisation s'exprime, attendu qu'elle ne se limite pas seulement à des productions économiques, mais aussi de sens. Les modes d'expression à travers lesquels la religion se redéfinit depuis quelques décennies en offrent d'éloquentes illustrations : les florissantes religiosités hybrides participent à la diffusion planétaire d'instruments d'homogénéisation tout en fortifiant les sacralités locales ${ }^{33}$.

Modernité, religiosité et sécularisme sont des processus, par définition actifs et organisés dans le temps, qui méritent d'être traités dans leur contextualité et leur mouvance. Il se trouve que les trois paradigmes cohabitent à la faveur de l'ascension des organisations transnationales qui, suite à la défaillance des grandes institutions du sens, héritent du pouvoir de déterminer la nature de l'ordre moral : les ONG. Produit hybride autant que spectaculaire, l'humanitaire revêt les attributs d'une dévotion civile. Les gourous flottants ne s'y contentent pas de seconds rôles.

Un nombre croissant d'enquêtes pertinentes nous apprend que l'essor de l'humanitarisme confessionnel est mondial ${ }^{34}$. En Inde, la voie de la bhakti peut habilement faire figure de dernier rempart contre une éventuelle désaffection vis-à-vis du religieux. Dévotion et renoncement ne désignent plus seulement, la première une activité rituelle ou une disposition psychique, le second un retrait de l'individu de ses obligations sociales et plus largement des lois de causalité. Grâce à des appareils conceptuels très librement inspirés des textes classiques, les deux attitudes passent par le filtre de la rationalisation pour être standardisées, et devenir des valeurs universelles modernes. De plus en plus, aspiration spirituelle et vocation humanitaire se confondent, au nom d'un idéal de la dévotion et du service synonyme de dévouement. S’investir dans le renoncement (!) est devenu le signe des esprits progressistes.

Pascale LÉPINASSE

EHESS

31. Chantal SAINT-BLANCAT, "Globalisation, réseaux et diasporas dans le champ religieux ", in La globalisation du religieux, op. cit., p. 77.

32. James Beckford parle de la religion comme d'un «bon analyseur des processus d'internationalisation, de transnationalisation et de globalisation ", "Perspectives sociologiques sur les relations entre modernité et globalisation religieuse ", in La globalisation du religieux, op. cit., p. 281.

33. S'ajoutant par conséquent à ceux déjà en usage, comme la mode vestimentaire, les codes publicitaires ou encore les systématismes linguistiques. Maya Warrier recommande de même d'envisager la relation au gourou moderne dans le cadre d'une dialectique entre global et local, universel et particulier, Hindu Selves in a Modern World. Guru Faith in the Mata Amritanandamayi, op. cit., p. 11.

34. Voir notamment, Sébastien FATH, Dieu bénisse l'Amérique. La religion de la Maison Blanche, Paris, Seuil, 2004 ; Jean-Pierre Berthon, Anne Bouchy et Pierre F. Souyri, Identités, marges, médiations. Regards croisés sur la société japonaise, Paris, EFEO, 2001 ; Abdel-Rahman GHANDOuR, Jihad humanitaire. Enquête sur les ONG islamiques, Paris, Flammarion, 2002. 


\section{Résumé}

Bien que le substrat hindou ne privilégie aucunement la philanthropie, les "gourous modernes " indiens les plus en vue bâtissent leur carrière de leaders charismatiques sur l'humanitaire. Auteurs d'hybridations audacieuses entre fondements brahmaniques et valeurs modernes, ils convertissent leurs mouvements sectaires en ONG transnationales, hautement plébiscitées par les nouvelles classes consuméristes indiennes. Dans une société assumant que les hommes ne naissent pas égaux, comment s'opère le passage d'une hiérarchie normative certes déclinante, mais âgée de plusieurs millénaires, à l'application d'une solidarité "événementielle»?

\section{Abstract}

This article deals with one of the salient features of contemporary Hinduism, that is to say the visibility of "modern gurus". These charismatic leaders, who preside over spiritual mega-congregations, specifically target consumerist classes and address them with the rhetoric of progressivism. They put an emphasis on an intellectual approach of the religious sphere as well as on social performance, whilst assigning themselves a large-scale educational mission. In support of their sustained proselytism, they divert local and deeply embedded religious attitudes from their original meaning, e.g. devotion (bhakti) or divine service (seva), to turn them into universal moral values. This instrumentalisation enables them to benefit from the status of an NGO, and this is the source of their transnational expansion.

\section{Resumen}

Aunque el sustrato bindú no privilegie de ninguna manera la filantropía, los "gurús modernos" indios más célebres construyen su carrera de líderes carismáticos sobre lo bumanitario. Autores de hibridaciones audaces entre fundamentos brabmánicos y valores modernos, convierten sus movimientos sectarios en ONG transnacionales, altamente plebiscitadas por las nuevas clases consumidoras indias. En una sociedad que asume que los hombres no nacen iguales, ¿ de qué maneras se opera el paisaje de una jerarquía normativa, por cierto declinante, pero de varios milenios de antigüedad, a la aplicación de una solidaridad "de los acontecimientos"? 
